\title{
ON THE EMBEDDABILITY AND NONEMBEDDABILITY OF CERTAIN PARALLELIZABLE MANIFOLDS ${ }^{1}$
}

\author{
BY W. C. HSIANG AND R. H. SZCZARBA
}

Communicated by W. S. Massey, February 25, 1963

Introduction. The problem of proving nonembeddability results for differentiable manifolds has received much attention in recent years. However, with few exceptions (see, for example Hantzsche [3] and Massey [8]), the techniques used require that the tangent bundle of the manifold in question be nontrivial; thus they do not apply to parallelizable manifolds. In this note, we study the embeddability of a certain sequence of parallelizable manifolds. As a consequence, we are able to show that for any positive integer $k$, there are parallelizable manifolds which do not embed with codimension ${ }^{2} k$. In addition, we give an example of a 22-dimensional manifold $M_{1}$ with the property that, for any $j, 1 \leqq j \leqq 7$, there are two embeddings of $M_{1}$ in $R^{30+j}$ with fiber homotopically distinct normal sphere bundles. ${ }^{3}$

The results announced in this note follow from a detailed study of the embeddability and nonembeddability of sphere bundles over spheres. The complete proofs will appear in a subsequent paper.

The authors would like to express their gratitude to W. S. Massey for suggesting the problem and for many stimulating conversations during the preparation of this paper.

Statement of results. In what follows, all manifolds, embeddings and immersions will be $C^{\infty}$ differentiable. We denote by $\tau(M)$ the tangent bundle of $M$ and by $\theta^{r}=\theta^{r}(M)$ the trivial $r$-plane bundle over $M$. If $\xi$ is a $(k-1)$-sphere bundle, $\xi$ will denote the associated $k$-plane bundle.

Let $S^{n-1}$ denote the $(n-1)$-sphere where $n=2^{4 q}, q \geqq 1$. It follows from results of Eckmann [2] and Adams [1] that $S^{n-1}$ has exactly $8 q$ independent vector fields. Thus we can find an $(n-8 q-1)$-sphere bundle $\xi_{q}$ over $S^{n-1}$ with a cross section and with the property that $\xi_{q} \oplus \theta^{8 q-1}=\tau\left(S^{n-1}\right)$. Let $M_{q}$ denote the total space of $\xi_{q}$. Clearly the dimension of $M_{q}$ is $2 n-8 q-2=2^{4 q+1}-8 q-2$.

The following proposition follows from Theorem IX of Kervaire

\footnotetext{
${ }^{1}$ During the preparation of this paper, both authors were partially supported by NSF grant number NSF-G-18995.

2 We say that $M$ embeds in Euclidean space with codimension $k$ if $M$ can be embedded in $R^{n+k}$ where $n=$ dimension $M$.

${ }^{3} \mathrm{~A}$. Haefliger has proved the existence of an embedding of $S^{11}$ in $R^{17}$ with a normal sphere bundle which is not trivial but is fiber homotopically trivial (see Massey [9]).
} 
[6] (see also Sutherland [12]).

Proposition. The manifolds $M_{q}$ are parallelizable.

Let $\zeta$ be the sphere bundle associated with $\hat{\xi}_{q} \oplus \theta^{8 q}$ and let $E_{\zeta}$ be its total space. Then $M_{q}$ can be embedded in $E_{\zeta}$. Furthermore, since $\hat{\xi}_{q} \oplus \theta^{8 q-1}=\tau\left(S^{n-1}\right), \zeta$ is the trivial $(n-1)$-sphere bundle over $S^{n-1}$ so $E_{\zeta}=S^{n-1} \times S^{n-1}$. Therefore $E_{\zeta}$ and consequently $M_{q}$ can be embedded in $R^{2 n-1}$. In fact, we prove

Theorem 1. For $q>1, M_{q}$ can be embedded in Euclidean space with codimension $8 q+1$ but not with codimension $8 q$.

To show that $M_{q}$ cannot be embedded with codimension $8 q$, we first prove that we can choose a cross section $s: S^{n-1} \rightarrow M_{q}$ of $\xi_{q}$ which is an embedding. In fact, we can pick $s$ to have a normal bundle $\nu_{s}$ with the property that $\nu_{s} \oplus \theta^{1}=\hat{\xi}_{q}$. Now suppose $M_{q}$ embeds in $R^{2 n-2}$ with normal bundle $\nu$. Using the fact that the composite embedding

$$
S^{n-1} \rightarrow M^{q} \rightarrow R^{2 n-2}
$$

has a trivial normal bundle (see Kervaire [7]), and the fact that $\nu_{s} \oplus \theta^{1}=\xi_{q}$, we prove that $\left(\nu \mid S^{n-1}\right) \oplus \theta^{n-8 q-2}=\tau\left(S^{n-1}\right)$ where $\nu \mid S^{n-1}$ is the restriction of $\nu$ to $s\left(S^{n-1}\right)$. However, this is impossible by the result of Adams [1] since $n-8 q-2>8 q$.

As an immediate consequence of Theorem 1, we have

COROLlary 1. For any positive integer $k$, there are parallelizable manifolds which cannot be embedded in Euclidean space with codimension $k$.

Following Sanderson [11], we define the divergence of a manifold $M$ to be $k-r$ where $k$ is the least integer such that $M$ can be embedded with codimension $k$ and $r$ is the least integer such that $M$ can be immersed with codimension $r$. Since any parallelizable manifold can be immersed with codimension 1 (see Hirsch [4]), we have

COROLlaRy 2. For any integer $k$, there are manifolds with divergence exceeding $k$.

We now turn our attention to the 22-dimensional manifold $M_{1}$.

TheOREM 2. The manifold $M_{1}$ can be embedded in $R^{30}$ but not in $R^{28}$. Furthermore, any embedding of $M_{1}$ in $R^{30}$ has a normal sphere bundle which is not fiber homotopically trivial.

We are unable to decide whether or not $M_{1}$ embeds in $R^{29}$.

The techniques used to prove Theorem 1 show that $M_{1}$ cannot be 
embedded in $R^{28}$ while the methods of James-Whitehead [5] are used to prove that any embedding of $M_{1}$ in $R^{30}$ must have a normal sphere bundle which is not fiber homotopically trivial. To show that $M_{1}$ does embed in $R^{30}$, we prove that there is a 6 -sphere bundle $\eta$ over $S^{15}$ with $\hat{\xi} \oplus \hat{\eta}=\theta^{15}$. Thus $M_{1}$ can be embedded in $S^{15} \times S^{14}$ which can be embedded in $R^{30}$.

In fact, it can be shown that $\hat{\eta} \oplus \theta^{8}=\tau\left(S^{15}\right)$ and that $\nu \mid S^{15}=\hat{\eta} \oplus \theta^{1}$ where $\nu$ is the normal bundle of the embedding of $M_{1}$ in $R^{30}$ described above. Therefore, if we consider the composite embedding $M_{1} \subset R^{30}$ $C R^{37}$, its normal bundle when restricted to $S^{15}$ is $\hat{\eta} \oplus \theta^{8}=\tau\left(S^{15}\right)$ which is not fiber homotopically trivial (see Milnor-Spanier [10, Theorem 2]). Furthermore, the embedding of $M_{1}$ in $R^{31}$ described just before the statement of Theorem 1 has a trivial normal bundle. Thus we have

THEOREM 3. For each $j, 1 \leqq j \leqq 7$, there are two embeddings of $M_{1}$ in $R^{30+j}$ with the property that the normal sphere bundle of the first is trivial while the normal sphere bundle of the second is not even fiber homotopically trivial.

\section{BIBLIOGRAPHY}

1. J. F. Adams, Vector fields on spheres, Ann. of Math. (2) 75 (1962), 603-632.

2. B. Eckmann, Gruppen theoretischer Beweis des Satzes von Hurwitz-Radon uber die Komposition quadratischer Formen, Comment. Math. Helv. 15 (1942), 358-366.

3. W. Hantzsche, Einlagerungen von Mannigfaltigkeiten in euklidische Ruume, Math. Z. 43 (1938), 38-55.

4. M. W. Hirsch, Immersions of manifolds, Trans. Amer. Math. Soc. 93 (1959), 242-276.

5. I. M. James and J. H. C. Whitehead, The homotopy theory of sphere bundles over spheres. I, Proc. London Math. Soc. (3) 4 (1954), 196-218.

6. M. Kervaire, Courbure integral generalisee et homotopie, Math. Ann. 131 (1956), 219-252.

7. - An interpretation of $G$. Whitehead's generalization of H. Hopf's invariant, Ann. of Math. (2) 69 (1959), 345-364.

8. W. S. Massey, On the cohomology ring of a sphere bundle, J. Math. Mech.7 (1958), 265-290.

9. - On the normal bundle of a sphere embedded in Euclidean space, Proc. Amer. Math. Soc. 10 (1959), 959-964.

10. J. Milnor and E. Spanier, Two remarks on fiber homotopy type, Pacific J. Math. 10 (1960), 585-590.

11. B. J. Sanderson, Immersions and embeddings of projective spaces (to appear).

12. W. Sutherland, $A$ note on the parallelizability of sphere bundles over spheres (to appear).

Princeton UnIVersity AND

YALE UNIVERSITY 